\section{P118 SHOULD WE TREAT OR RESCREEN PATIENTS FIRST WITH EQUIVOCAL CHLAMYDIA AND GONORRHOEA NAAT RESULTS?}

${ }^{1}$ Katie Ovens*, ${ }^{2}$ Erasmus Smit, ${ }^{1}$ Sarah Barrett. ${ }^{1}$ Birmingham Heartlands Hospital, Birmingham, UK; ${ }^{2}$ PHE, Birmingham Laboratory, Heartlands Hospital, Birmingham, UK

10.1136/sextrans-2015-052126.161

Background/introduction Equivocal NAAT results for Chlamydia and gonorrhoea (GC) cause treatment dilemmas for health professionals as there are no definitive management guidelines. Debate continues whether to rescreen and treat patients with equivocal results or rescreen the patient and await results before treatment.

Aim(s)/objectives To investigate rescreening tests for equivocal results and establish when patients should be offered treatment.

Methods A retrospective study of equivocal results from 2 GUM clinics between November 2013 and May 2014, and a third clinic between March 2010 and May 2014. HIV positive patients' results were included. Paper notes or electronic systems were examined. Data was collected using a standardised proforma and analysed using excel software.

Results 76 equivocal results $(2.2 \%$ of positive results) were investigated. 62 patients $(83.8 \%)$ attended recall appointments, 36 patients $(58.0 \%)$ were offered treatment and rescreened, $14(22.6 \%)$ were rescreened and awaited results prior to treatment and 2 patients $(3.2 \%)$ were treated with no retest sent. 8 patients $(16.1 \%)$ were treated due to a positive GC result at a second site alongside the equivocal result. Of the 54 equivocal results re-tested, $3(5.6 \%)$ were positive and all of these resulted from equivocal GC tests (19 rescreened). All 35 rescreened equivocal Chlamydia tests were negative.

Discussion/conclusion There is currently variation in how clinicians are managing equivocal results. The findings suggest that initiating treatment for Chlamydia before rescreening may result in over treatment. GC equivocal results are more likely to be positive on re-testing, thus clinicians should have a lower threshold for treating these at the time of rescreening.

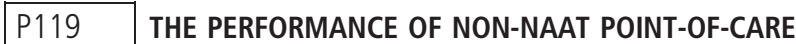 (POC) TESTS AND RAPID-NAAT TESTS FOR CHLAMYDIA AND GONORRHOEA INFECTIONS. AN ASSESSMENT OF CURRENTLY AVAILABLE ASSAYS}

Gary Brook*. Central Middlesex Hospital, London North West Healthcare NHS Trust, London, UK

\subsection{6/sextrans-2015-052126.162}

Objectives To identify POC and rapid-NAATs for the diagnosis of chlamydia and gonorrhoea and assess their utility.

Methods Literature search for available POC and rapid-NAATs. The performance from the best-performing assays were applied hypothetically to patients in this clinic in which 100 consecutive patients with chlamydia and 100 with gonorrhoea were diagnosed in 1737 and 4575 patients respectively, with 44/100 and $54 / 100$ treated at first attendance respectively.

Results 11 POC and 1 rapid-NAAT identified. Published performances for the best POC for chlamydia were: sensitivity $41-87 \%$, specificity $89-99.6 \%$. Our data suggest that if this assay were used instead of our current NAAT, for every 100 patients diagnosed currently, 23-46 extra patients would be treated at first attendance; 10-35 would go undiagnosed with 7-191 false-positives. Best chlamydia rapid-NAAT: sensitivity
97.5-98.7\%, specificity 99.4-99.9\%. Anticipated performance for every 100 patients diagnosed currently: 0 extra patients treated at first attendance, 1-3 undiagnosed, 0-2 false-positives. Best POC for gonorrhoea: sensitivity 54-70\%, specificity 97-98\%. Anticipated performance for every 100 patients diagnosed currently: 14-18 extra patients treated at first attendance, 28-32 undiagnosed, 92-137 false-positives. Best rapid-NAAT for gonorrhoea: sensitivity $96-100 \%$, specificity $99.9-100 \%$. Anticipated performance for every 100 patients diagnosed currently: 0 extra patients treated at first attendance, $0-4$ undiagnosed, 0-5 false-positives. Rapid-NAAT would reduce time to treatment by 4 days.

Conclusion POC assays would need to be used in conjunction with a NAAT, increasing early treatment rates, expense and false-positive results. The rapid-NAAT could be used alone, with a reduction in average time-to-treat and a small reduction in sensitivity and specificity.

\section{P120 INJECTING, OBESITY AND ANTIBIOTIC RESISTANCE: AN EXPLORATION OF NURSING PRACTICE IN RELATION TO THE ADMINISTRATION OF INTRAMUSCULAR INJECTION IN THE TREATMENT OF GONORRHOEA}

${ }^{1}$ Caroline Donnelly*, ${ }^{2}$ Sara MacDonald. 'Sandyford Sexual Health Services, NHS Greater Glasgow \& Clyde, Glasgow, UK; ${ }^{2}$ University of Glasgow, Glasgow, UK

\subsection{6/sextrans-2015-052126.163}

Background/introduction Traditionally, conventional green needles (1.5 inch) are used to reach the dorsogluteal muscle. However, in the face of increasing obesity, there may be difficulty in reaching the target muscle due to subcutaneous fat. This can lead to potential ineffective delivery of medication resulting in non-treatment of infection, and possibly contributing to antibiotic resistance.

Aim(s)/objectives To explore existing practice of sexual health practitioners in relation to site and technique when administering intramuscular gonorrhoea treatment, in NHS Greater Glasgow and Clyde.

Methods Focus group interviews with 22 sexual health participants with a variety of experiences. Interviews were analysed using a framework approach.

Results

- The dorsogluteal muscle was used for all injections excluding vaccines.

- Only two participants had heard of the recommended ventrogluteal site.

- Mentors were key influences in role modelling within clinical situations.

- No updates were reported since learning this basic skill as a student.

Despite awareness of the obesity epidemic, using a longer needle or changing target muscle site had not been contemplated until the focus group.

Discussion/conclusion Obesity constitutes health challenges to basic nursing care, and commands a practical skilled workforce in anticipation of these complexities. This study reveals a theorypractice gap in the essential assessment of appropriate target muscle, which has potential to compound resistance issues. As rapid emergence of resistant strains pose a threat to untreatable gonorrhoea, we recommend that adoption of best practice guidance is essential alongside further study to ensure efficacy of treatment. 